\title{
The List-Chromatic Number of Infinite Graphs Defined on Euclidean Spaces
}

\author{
Péter Komjáth
}

Received: 14 September 2009 / Revised: 1 October 2009 / Accepted: 2 October 2009 /

Published online: 22 October 2009

(C) Springer Science+Business Media, LLC 2009

\begin{abstract}
If $D$ is a countable set of positive reals, $2 \leq n<\omega$, let $X_{n}(D)$ be the graph with the points of $\mathbf{R}^{n}$ as vertices where two vertices are joined iff their distance is in $D$. We determine the list-chromatic number of $X_{n}(D)$ as much as possible.
\end{abstract}

Keywords List-chromatic number - Coloring number - Graphs defined by distances $\cdot$ Euclidean space $\cdot$ Independence proofs

If $D$ is a countable set of positive numbers, then let $X_{n}(D)$ denote the $n$-dimensional $D$-distance graph. That is, the vertex set is $\mathbf{R}^{n}$, and two points are joined if their distance is in $D$.

In this paper we make some remarks on $\operatorname{List}\left(X_{n}(D)\right)$ for certain values of $D$ and $n$, where for a graph $X, \operatorname{Chr}(X)$ denotes the chromatic number, $\operatorname{Col}(X)$ the coloring number, and $\operatorname{List}(X)$ the list-chromatic number of $X$. The inequality $\operatorname{Chr}(X) \leq \operatorname{List}(X) \leq \operatorname{Col}(X)$ immediately connects these notions. In general, $\operatorname{Chr}(X)$ and $\operatorname{Col}(X)$ can be far away; there are bipartite graphs with large coloring number. Namely, $\operatorname{Col}(K(\kappa, \kappa))=\kappa$ and $\operatorname{Col}(K(\kappa, \lambda))=\kappa^{+}$when $\kappa<\lambda$ are infinite.

The results of [8] show that the list-chromatic number can be close to the chromatic number and also to the coloring number, depending on the model of set theory. We determine $\operatorname{List}\left(X_{n}(D)\right)$ as much as it is possible. Namely, $\operatorname{List}\left(X_{2}(D)\right)=\aleph_{0}$ always holds, $\operatorname{List}\left(X_{3}(D)\right)$ is countable if $D$ is finite or is a sequence converging to 0 and is $\aleph_{1}$ otherwise, $\aleph_{1} \leq \operatorname{List}\left(X_{n}(D)\right) \leq c(4 \leq n<\omega)$ and, depending on the model of set theory, can be either of the two extrema.

Communicated by Imre Bárány.

Research partially supported by Hungarian National Research Grant K 81121.

P. Komjáth $(\bowtie)$

Department of Computer Science, Eötvös University, P.O. Box 120, Budapest 1518, Hungary

e-mail: kope@cs.elte.hu 
History A seminal result of Erdős and Hajnal on infinite graphs states that if $(V, X)$ is an infinite graph that does not contain $K\left(n, \aleph_{1}\right)$ as subgraph for some finite $n$, then $\operatorname{Col}(V, X) \leq \aleph_{0}$ (Corollary 5.6, [4], p. 72).

The first theorem in the topic we are interested in was given by Erdôs [3], who noticed that as $\mathbf{Q}$ is countable, $X_{2}(\mathbf{Q})$ is easily seen not to contain the bipartite graph $K\left(2, \aleph_{1}\right)$, and so $\operatorname{Col}\left(X_{2}(\mathbf{Q})\right) \leq \aleph_{0}$ by the above result.

The question whether $\operatorname{Chr}\left(X_{n}(\mathbf{Q})\right) \leq \aleph_{0}$ for every $n$ became one of the problems Erdős liked to mention. We notice that the above Erdős-Hajnal proof worked for any countable $D$; in fact, the results in this topic were formulated for specific countable sets but worked for the general case, see our reformulated versions below.

Notice also that if $D$ is finite, then each $\operatorname{Chr}\left(X_{n}(D)\right)$ is finite. Finding, however, the correct value is a notoriously hard problem even for $D=\{1\}$.

In [5] we proved that $\operatorname{Chr}\left(X_{3}(\mathbf{Q})\right) \leq \aleph_{0}$ and noticed that $\operatorname{Col}\left(X_{3}(\mathbf{Q})\right)>\aleph_{0}$. The argument for the latter inequality actually works for any countably infinite $D$ unless it is a sequence converging to zero, see Lemma 2 below. The full conjecture of Erdôs was eventually proved in [7]. Later Schmerl gave a very short proof of a stronger statement (Theorem 4 below, for a proof, see [9]).

Jensen and Toft [6] remarked that a result of Alon [1] implies that $\operatorname{List}\left(X_{2}(1)\right)$ is infinite and asked whether $\operatorname{List}\left(X_{2}(1)\right)=\aleph_{0}$. As we saw above, this had been answered, although not explicitly, much earlier. Schmerl [10] proved that $\operatorname{List}\left(X_{2}(1)\right)=\aleph_{0}$ and even $\operatorname{List}\left(X_{3}(1)\right)=\aleph_{0}$, and remarked that $\operatorname{List}\left(X_{4}(1)\right)>\aleph_{0}$ and $\operatorname{List}\left(X_{2}(\mathbf{Q})\right)=\aleph_{0}<\operatorname{List}\left(X_{3}(\mathbf{Q})\right)$.

The recent paper [2] rediscovered some of the above results, namely, $\operatorname{List}\left(X_{2}(\mathrm{ODD})\right)=\aleph_{0}<\operatorname{List}\left(X_{3}(\mathrm{ODD})\right)$, where ODD denotes the set of odd natural numbers. The authors of [2] were primarily interested in the fascinating, and apparently hard, conjecture that $\operatorname{Chr}\left(X_{2}(\mathrm{ODD})\right)$ is finite.

Definitions $\mathbf{Q}$ is the set of rationals, and $\mathbf{R}$ is the set of reals. $c=|\mathbf{R}|$ is the cardinal continuum. If $S$ is a set, $\kappa$ a cardinal, then we define $[S]^{\kappa}=\{X \subseteq S:|X|=\kappa\}$ and $[S]^{<\kappa}=\{X \subseteq S:|X|<\kappa\}$. A $\operatorname{graph}(V, X)$ is any pair where $V$ is a set (the vertices) and $X \subseteq[V]^{2}$ (the edges). $K(\kappa, \lambda)$ denotes the complete bipartite graph with bipartition classes of cardinalities $\kappa, \lambda$, respectively. A good coloring of a graph $(V, X)$ is any function on $V$ such that $f(x) \neq f(y)$ whenever $\{x, y\} \in X$. The chromatic number $\operatorname{Chr}(V, X)$ or $\operatorname{Chr}(X)$ of a graph $(V, X)$ is the least cardinal $\kappa$ such that there is a good coloring $f: V \rightarrow \kappa$. If $\kappa$ is a cardinal, the graph $(V, X)$ is $\kappa$-choosable if for every assignment $F$ with $|F(x)|=\kappa$, there is a choice function $f(x) \in F(x)$ which is a good coloring of $(V, X)$. The list-chromatic number $\operatorname{List}(V, X)$ or $\operatorname{List}(X)$ of a graph $(V, X)$ is the least cardinal $\kappa$ such that $(V, X)$ is $\kappa$-choosable. The coloring number $\operatorname{Col}(V, X)$ or $\operatorname{Col}(X)$ of a graph $(V, X)$ is the smallest cardinal $\mu$ such that there is a well-ordering $\prec$ of the vertex set $V$ such that $|\{y \prec x:\{y, x\} \in X\}|<\mu$ for every $x \in V$.

Lemma $1 \operatorname{Chr}(V, X) \leq \operatorname{List}(V, X) \leq \operatorname{Col}(V, X) \leq|V|$ for every graph $(V, X)$.

Proof For the first inequality, assume that $(V, X)$ is $\kappa$-choosable. Then specifically, there is a good coloring $f(x) \in F(x)$, where $F(x)=\kappa$ for every $x \in V$, and this establishes $\operatorname{Chr}(V, X) \leq \kappa$. 
For the second inequality, assume that $\operatorname{Col}(V, X) \leq \kappa$ for some cardinal. This means that there is a well-ordering $\prec$ of the vertex set $V$ such that $\mid\{y \prec x:\{y, x\} \in$ $X\} \mid<\kappa$ for every $x \in V$. If $F$ is a function with $|F(x)|=\kappa$ for every $x \in V$, then we select a good coloring $f(x) \in F(x)$ by transfinite recursion on $\prec$ : let $f(x)$ be an arbitrary element of the set $F(x)-\{f(y): y \prec x,\{y, x\} \in X\}$. The latter set is nonempty as $|F(x)|=\kappa$, and we subtract a smaller set. It is clear that $f$ is, indeed, a good coloring.

For proving the last inequality, it suffices to consider any well-ordering into the order type $|V|$. Recall that in axiomatic set theory, $|V|$, the cardinality of $V$, is identified with the smallest ordinal which is equicardinal to $V$, so a well-ordering into order type $|V|$ is one where each element is preceded by less than $|V|$ elements.

Lemma $2([2,5,10])$ If $D$ is infinite and is not a sequence converging to 0 , then for $n \geq 3, X_{n}(D)$ contains a $K\left(\aleph_{0}, c\right)$. If $D \neq \emptyset$, then for $n \geq 4, X_{n}(D)$ contains $a$ $K(c, c)$.

Proof In the first statement we can assume that $n=3$. Choose distinct elements $d_{0}, d_{1}, \ldots \in D$ such that $d_{i}>k(i<\omega)$ for some $k>0$. Set $A=\left\{p_{0}, p_{1}, \ldots\right\}$ where $p_{i}=\left(0,0, \sqrt{d_{i}^{2}-k^{2}}\right)$, and let the elements of $B$ be the points of the form $(x, y, 0)$ with $x^{2}+y^{2}=k^{2}$, i.e., $B$ is a full circle. The distance of $p_{i}$ from any element of $B$ is $\sqrt{d_{i}^{2}-k^{2}+k^{2}}=d_{i}$.

For the second statement, we can assume that $D=\{d\}$ and $n=4$. Then $A$ and $B$ can be chosen as orthogonal circles:

$$
A=\left\{(x, y, 0,0): x^{2}+y^{2}=\frac{d^{2}}{2}\right\}
$$

and

$$
B=\left\{(0,0, z, u): z^{2}+u^{2}=\frac{d^{2}}{2}\right\} .
$$

Obviously, the distance between a point of $A$ and a point of $B$ is $d$.

Lemma 3 If $\kappa$ is an infinite cardinal, then $\operatorname{List}\left(K\left(\kappa, 2^{\kappa}\right)\right)>\kappa$.

Proof Let $(V, X)$ be the complete bipartite graph with bipartition classes $A$ and $B$ such that $|A|=\kappa$ and $|B|=2^{\kappa}$. We are going to give an assignment $F$ with $|F(x)|=$ $\kappa$ for $x \in V$ such that no choice function $f \in \prod\{F(x): x \in V\}$ is a good coloring of $(V, X)$. First, let $\{F(x): x \in A\}$ be an arbitrary system of pairwise disjoint $\kappa$-size sets. Then, for every choice function $g \in \prod\{F(x): x \in A\}$, select a $y_{g} \in B$ and make $F\left(y_{g}\right)=\{g(x): x \in A\}$. As the sets $\{F(x): x \in A\}$ are disjoint, each $F\left(y_{g}\right)$ is of cardinality $\kappa$. The number of choice functions is $\left|\prod\{F(x): x \in A\}\right|=\kappa^{\kappa}=2^{\kappa}=$ $|B|$; therefore, it is possible to choose a different $y_{g}$ for each choice function $g$.

Assume now that some $f \in \prod\{F(x): x \in V\}$ is a good coloring of $(V, X)$. Set $g=f \mid A$. Then $f\left(y_{g}\right) \in F\left(y_{g}\right)=\{f(x): x \in A\}$, and so $f\left(y_{g}\right)=f(x)$ for some $x \in A$, which shows that $f$ is not a good coloring, as $x$ and $y_{g}$ are joined. 
Theorem 4 (J. Schmerl) If D is any countable set of distances, then there is a wellordering $\prec$ of $\mathbf{R}^{n}$ such that $\inf \{d(y, x): y \prec x, d(y, x) \in D\}>0$ for every $x \in \mathbf{R}^{n}$.

Corollary 5 (Komjáth [7]) $\operatorname{Chr}\left(X_{n}(D)\right) \leq \aleph_{0}$ for every countable $D$ and any $n<\omega$.

Proof Set $\delta(x)=\inf \{d(y, x): y \prec x, d(y, x) \in D\}$. We color $\mathbf{R}^{n}$ as follows. For $x \in \mathbf{R}^{n}$, set $f(x)=(q, k)$, where $q \in \mathbf{Q}^{n}$ is a point with rational coordinates, and $k$ is a natural number such that $d(q, x)<\frac{1}{2 k}$ and $\delta(x)>\frac{1}{k}$. This is a coloring with countably many colors. In order to show the required property of the coloring, assume that $f(x)=f(y)=(q, k)$. Without loss of generality, $y \prec x$, but then, as $d(y, x) \leq d(y, q)+d(q, x)<\frac{1}{2 k}+\frac{1}{2 k}=\frac{1}{k}<\delta(x)$, we get that $d(y, x) \notin D$, as desired.

Theorem 6 (Schmerl [10]) If D is infinite and not a sequence converging to zero, then $\operatorname{List}\left(X_{3}(D)\right) \geq \aleph_{1}$.

Proof By Lemma 2, $X_{3}(D)$ contains a $K\left(\aleph_{0}, c\right)$ whose list-chromatic number is at least $\aleph_{1}$, by Lemma 3 .

Theorem 7 If $D$ is finite or a sequence converging to 0 , then $\operatorname{Col}\left(X_{3}(D)\right) \leq \aleph_{0}$. If $D$ is an arbitrary countable set, then $\operatorname{Col}\left(X_{3}(D)\right) \leq \aleph_{1}$.

Proof We give a unified proof of the two statements. We start with the following claim.

Claim For any infinite $Y \subseteq \mathbf{R}^{3}$, there is a unique smallest $Z$ with $Y \subseteq Z \subseteq \mathbf{R}^{3}$, $|Z|=|Y|$, such that

(a) If $x_{0}, x_{1}, x_{2} \in Z$ are not collinear and $y \in \mathbf{R}^{3}$ has $d\left(y, x_{i}\right) \in D(i<3)$, then $y \in Z$.

(b) If $y_{0}, y_{1} \in Z$, $z$ is on the connecting line of $y_{0}, y_{1}$, and $d\left(z, y_{0}\right) \in D$, then $z \in Z$.

Proof Given $x_{0}, x_{1}, x_{2}$ as in (a), let $f\left(x_{0}, x_{1}, x_{2}\right)$ be the set of possible $y$, and given $y_{0}, y_{1}$ as in (b), let $g\left(y_{0}, y_{1}\right)$ be the set of possible $z$. Notice that $f\left(x_{0}, x_{1}, x_{2}\right)$ and $g\left(y_{0}, y_{1}\right)$ are always countable.

Set $Z=Z_{0} \cup Z_{1} \cup \cdots$, where $Z_{0}=Y$, and

$$
Z_{n+1}=Z_{n} \cup \bigcup\left\{f\left(x_{0}, x_{1}, x_{2}\right): x_{0}, x_{1}, x_{2} \in Z_{n}\right\} \cup \bigcup\left\{g\left(y_{0}, y_{1}\right): y_{0}, y_{1} \in Z_{n}\right\} .
$$

Then $Z$ is as required.

Call the above set the closure of $Y$ and denote it by $\operatorname{cl}(Y)$. We prove by transfinite induction on $\kappa=|Y|$ that every subset $Y \subseteq \mathbf{R}^{3}$ has a well-ordering as specified in the theorem. This is obvious if $\kappa$ is finite or $\aleph_{0}$. Assume that $\kappa>\aleph_{0}$ and we have the result for sets of smaller cardinality. If $|Y|=\kappa$, then we can decompose $Y$ as $Y=$ $\bigcup\left\{Y_{\alpha}: \alpha<\kappa\right\}$ into an increasing union of subsets with $Y_{0}=\emptyset, Y_{\alpha}=\operatorname{cl}\left(Y_{\alpha}\right),\left|Y_{\alpha}\right|<\kappa$ for $\alpha<\kappa$, and $Y_{\alpha}=\bigcup\left\{Y_{\beta}: \beta<\alpha\right\}$ whenever $\alpha<\kappa$ is a limit ordinal. For example, 
if $Y=\left\{y_{\alpha}: \alpha<\kappa\right\}$ is an enumeration of $Y$, we can have $Y_{\alpha}=\operatorname{cl}\left(\left\{y_{\beta}: \beta<\alpha\right\}\right)$ for $\alpha<\kappa$. As $\left|Y_{\alpha+1}-Y_{\alpha}\right| \leq\left|Y_{\alpha+1}\right|<\kappa$, we can apply the inductive hypothesis and obtain a well-ordering $\prec_{\alpha}$ of $Y_{\alpha+1}-Y_{\alpha}$ witnessing the (appropriate) statement of the theorem, i.e., the number of edges going down from any point is finite/countable. We define $\prec$ on $Y$ as follows. Set $x \prec y$ if either $x, y \in Y_{\alpha+1}-Y_{\alpha}$ for some $\alpha$ and $x \prec_{\alpha} y$ or else $x \in Y_{\alpha+1}-Y_{\alpha}, y \in Y_{\beta+1}-Y_{\beta}$, and $\alpha<\beta$. Clearly, $\prec$ is a well-ordering of $Y$. To show the required property we have to prove that if $x \in Y_{\alpha+1}-Y_{\alpha}$, then the number of edges from $x$ into $Y_{\alpha}$ is finite/countable. Let $Z \subseteq Y_{\alpha}$ be the set of the other endpoints, i.e., $z \in Z$ if $z \in Y_{\alpha}$ and $d(z, x) \in D$. $Z$ cannot contain three noncollinear points, as that would force $x \in Y_{\alpha}$, a contradiction. The elements of $Z$ therefore are all collinear, that is, $Z=Y_{\alpha} \cap L$ for some line $L$. Further, $x \notin L$, as otherwise we would get $x \in Y_{\alpha}$, a contradiction.

Now $Z=\{z \in L: d(x, z) \in D\}$ is countable, and it is finite if $D$ is a zero-sequence as $x \notin L$, so it is in positive distance from $L$.

Theorem 8 It is consistent that $c=\aleph_{2}$ and $\operatorname{List}\left(X_{n}(D)\right) \leq \aleph_{1}$ for every $n<\omega$ and countable D.

Proof We refer to the model of [8], where the following combinatorial principle holds.

(*) $c=\aleph_{2}$, and if $\left\{A_{\alpha}: \alpha<\omega_{2}\right\} \subseteq\left[\omega_{2}\right]^{\aleph_{1}}$, then there is a coloring $g: \omega_{2} \rightarrow \omega$ such that $g$ assumes every value on every $A_{\alpha}$.

Assume that $V=\mathbf{R}^{n}$ and $X=X_{n}(D)$. We already know from Corollary 5 that $\operatorname{Chr}(V, X) \leq \aleph_{0}$. It suffices therefore to deduce the following from $(*)$ : if $(V, X)$ is a countably chromatic graph of cardinality at most $c=\aleph_{2}$, then $\operatorname{List}(V, X) \leq \aleph_{1}$. For this, let $F(x)(x \in V)$ be an assignment with sets of cardinality $\aleph_{1}$. Without loss of generality, $V=\omega_{2}$ and $F(x) \in\left[\omega_{2}\right]^{\aleph_{1}}$. Let $g: \omega_{2} \rightarrow \omega$ be a coloring as in $(*)$ for the system $\left\{F(\alpha): \alpha<\omega_{2}\right\}$, and let $\omega_{2}=V_{0} \cup V_{1} \cup \cdots$ be a good coloring of $\left(\omega_{2}, X\right)$, i.e., all edges go between different $V_{i}$. Now, if $\alpha \in V_{i}$, then let $f(\alpha)$ be an element of $F(\alpha)$ such that $g(f(\alpha))=i$. This is possible, as $g$ assumes every value in $F(\alpha)$. $f$ is a good coloring: if $\{\alpha, \beta\} \in X$, then $\alpha \in V_{i}$ and $\beta \in V_{j}$ for some $i \neq j$, thus $g(f(\alpha))=i \neq j=g(f(\beta))$, and therefore $f(\alpha) \neq f(\beta)$.

Notice that similar arguments give that it is consistent that $c=\aleph_{3}$, or any greater cardinal, and the claim that $\operatorname{List}\left(X_{n}(D)\right) \leq \aleph_{1}$ for every $n<\omega$ and countable $D$ still holds.

Theorem 9 If $2^{\aleph_{0}}=2^{\aleph_{1}}=\aleph_{2}$, then $\operatorname{List}\left(X_{n}(D)\right)=c$ for every $D \neq \emptyset$ and $n \geq 4$.

Proof By Lemma 1, the list chromatic number of $X_{n}(D)$ is at most $c$. By Lemma 2, $K\left(\aleph_{1}, \aleph_{2}\right)$ embeds into $X_{n}(D)$. By Lemma 3 , the list chromatic number of $K\left(\aleph_{1}, \aleph_{2}\right)$ is greater than $\aleph_{1}$.

Of course, the same argument gives that if $2^{\kappa}=\kappa^{+}=c$ for some cardinal $\kappa$ or if $c$ is weakly inaccessible and $2^{\mu}=c$ for every cardinal $\mu<c$, then $\operatorname{List}\left(X_{n}(D)\right)=c$ when $D \neq \emptyset, n \geq 4$, i.e., $c$ can be arbitrarily large, and $\operatorname{List}\left(X_{n}(D)\right)=c$ may still hold. 
Acknowledgement The author is grateful to the referee whose suggestions have greatly improved the exposition.

\section{References}

1. Alon, N.: Restricted colorings of graphs. In: Walter, K. (ed.) Surveys in Combinatorics, Proc. 14th British Combinatorial Conference, pp. 1-33. Cambridge University Press, Cambridge (1993)

2. Ardal, H., Man̆uch, J., Rosenfeld, M., Shelah, S., Stacho, L.: The odd-distance plane graph. Discrete Comput. Geom. 42, 132-141 (2009)

3. Erdős, P.: Problems and results in chromatic number theory. In: Harary, F. (ed.) Proof Techniques in Graph Theory, pp. 47-55. Academic Press, San Diego (1969)

4. Erdős, P., Hajnal, A.: On chromatic number of graphs and set systems. Acta Math. Acad. Sci. Hung. 17, 61-99 (1966)

5. Erdős, P., Komjáth, P.: Countable decompositions of $\mathbf{R}^{2}$ and $\mathbf{R}^{3}$. Discrete Comput. Geom. 5, 325-331 (1990)

6. Jensen, T.R., Toft, B.: Graph Coloring Problems. Wiley, New York (1995)

7. Komjáth, P.: A decomposition theorem for $\mathbf{R}^{n}$. Proc. Am. Math. Soc. 120, 921-924 (1994)

8. Komjáth, P.: The list-chromatic number of infinite graphs. Manuscript

9. Komjáth, P., Totik, V.: Problems and Theorems in Classical Set Theory. Springer, Berlin (2006)

10. Schmerl, J.H.: The list-chromatic number of Euclidean space. Geombinatorics 5, 65-68 (1995) 